\title{
Endobronchial ultrasound guided Echo Tip Ultra HD 22 G needle versus Echo Tip Pro Core HD 22G needle in diagnosis of mediastinal lesions
}

Sabah Ahmed Hussein ${ }^{1 *}$, Alaa Eldein Omar Shalaby ${ }^{1}$, Khaled Mahmoud Kamel ${ }^{1}$, Amal Ahmad Hareedy ${ }^{2}$ and Hoda Mohamed Mahmoud Abdelhameed ${ }^{1}$

\begin{abstract}
Background: Convex probe endobronchial ultrasound is a minimally invasive technique used for performing realtime transbronchial needle aspiration of enlarged hilar and mediastinal lymph nodes and could be used for diagnosing proximal peribronchial lesions. Although computed tomography, magnetic resonant imaging, and positron emission tomography/computed tomography have relatively high sensitivity for mediastinal evaluation, pathology is the gold standard. This study compared between Echo Tip Ultra HD-EBUS 22G needle and Echo Tip ProCore HD-EBUS 22G needle in diagnosis of mediastinal lesions using convex probe endobronchial ultrasound.

Results: Twenty-four patients with mediastinal lesions were included. Convex probe endobronchial ultrasoundguided transbronchial needle aspiration using both Echo Tip Ultra HD 22G and Echo Tip ProCore HD 22G needles was done to all patients with no peripheral lymph node enlargement. CP-EBUS TBNA had diagnostic accuracy 50\%, sensitivity $47.6 \%$, and specificity $100 \%$ in diagnosis of mediastinal lesions. The Echo Tip Ultra HD needle had diagnostic accuracy $40.9 \%$, sensitivity $38 \%$, and specificity $100 \%$ compared to the Echo Tip ProCore HD needle which had diagnostic accuracy $31.8 \%$, sensitivity $82.5 \%$, and specificity $100 \%$. The difference between both needles was statistically insignificant. The Echo Tip Ultra 22G HD needle had more representative cellularity (82\%) compared to the Echo Tip ProCore 22G HD needle (45\%) with a significant difference between both needles ( $p$ value $=0.001$ ).

Conclusion: Both needles can be used equally in sampling mediastinal lesions. This study supports that Echo Tip Ultra HD and Echo Tip ProCore HD needles can be used equally in sampling of mediastinal lesions and supports ultrasound screening of enlarged supraclavicular and cervical lymph nodes before performing EBUS-TBNA in patients with mediastinal lesions.
\end{abstract}

Keywords: EBUS-TBNA, Echo Tip Ultra HD 22G EBUS needle, Echo Tip ProCore HD 22G EBUS needle, Mediastinal lesions

\section{Background}

Convex probe endobronchial ultrasound (CP-EBUS) is a minimally invasive technique used for performing realtime transbronchial needle aspiration (TBNA) of enlarged hilar, mediastinal lymph nodes, and proximal peribronchial lesions [1].

\footnotetext{
* Correspondence: Sabah.Hussein@kasralainy.edu.eg

${ }^{1}$ Chest Department, Faculty of Medicine, Cairo University, Giza, Egypt

Full list of author information is available at the end of the article
}

For mediastinal evaluation, a variety of techniques are available: bronchoscopy, CT (computed tomography), MRI (magnetic resonance imaging), PET (positron emission tomography), and mediastinoscopy [2].

Although CT, MRI, and PET/CT have high sensitivity for mediastinal evaluation, pathology is the gold standard for diagnosis. Mediastinoscopy is the gold standard for the diagnosis of mediastinal disease. However, it has intraoperative and post-surgical risks and has limited

\section{Springer Open}

(- The Author(s). 2020 Open Access This article is licensed under a Creative Commons Attribution 4.0 International License, which permits use, sharing, adaptation, distribution and reproduction in any medium or format, as long as you give appropriate credit to the original author(s) and the source, provide a link to the Creative Commons licence, and indicate if changes were made. The images or other third party material in this article are included in the article's Creative Commons licence, unless indicated otherwise in a credit line to the material. If material is not included in the article's Creative Commons licence and your intended use is not permitted by statutory regulation or exceeds the permitted use, you will need to obtain permission directly from the copyright holder. To view a copy of this licence, visit http://creativecommons.org/licenses/by/4.0/. 
ability to evaluate hilar nodes. Consequently, all these factors contribute to the limited applicability for mediastinal evaluation [3].

EBUS-TBNA combined with endoscopic ultrasound fine needle aspiration (EUS-FNA) has replaced surgical staging as the initial test of choice for mediastinal evaluation [4].

This study compared between Echo Tip Ultra HDEBUS 22G and Echo Tip ProCore HD-EBUS 22G needles in diagnosis of mediastinal lesions using CP-EBUS.

\section{Methods}

This prospective study included 24 patients with mediastinal lesions, conducted in the Chest Department in collaboration with the Cytopathology Department, Faculty of Medicine, Cairo University Hospitals, during the period from January 2016 to August 2017. The Research Ethics Committee, Faculty of Medicine, Cairo University, approved this study. All patients above 18 years with no sex predilection presenting with mediastinal lesions were included. Patients with refractory hypoxia, hemodynamic instability, coagulopathy, recent myocardial infarction, or ischemia were excluded. Also, patients with endobronchial lesion suitable for bronchoscopic biopsy or enlarged palpable peripheral lymph node were excluded.

All patients were subjected to history taking, clinical examination, and laboratory investigations, e.g. complete blood picture, coagulation profile, renal and liver function tests, tuberculin skin test, and CT chest with IV contrast. CP-EBUS-guided TBNA using both CP-EBUSguided Echo Tip Ultra HD 22G and Echo Tip ProCore HD $22 \mathrm{G}$ needles was done to all patients with no peripheral lymph node enlargement.

\section{EBUS-TBNA technique [5]}

The patient was informed about the indication, the risks, and the benefits of EBUS-TBNA procedure, and informed consent was taken. The patient fasted for $6 \mathrm{~h}$ before the procedure to minimize the risk of aspiration during sedation. During the procedure, the patient was monitored (heart rate, blood pressure, and pulse oximetry).

Pre-medication: $0.5-1 \mathrm{mg}$ atropine sulphate intramuscular was given half an hour before the procedure and $0.5 \mathrm{mg} / \mathrm{kg}$ intravenous propofol was administered to be titrated according to clinical response.

Topical Anesthesia: was used by applying a lidocaine solution (2\%) on the upper airways.

Steps: The patient was lying in the supine position and the operator was standing behind the patient's head. Ordinarily CP-EBUS bronchoscope was introduced transoral. After visualization of the vocal cords, $2 \mathrm{ml}$ lidocaine solution (2\%) was used to anaesthetize the vocal cords and upper trachea then CP-EBUS was flexed $45^{\circ}$ to facilitate its advancement beyond vocal cords and once advanced all secretions were suctioned. Consequently,
Table 1 Sensitivity, specificity, predictive value, and diagnostic accuracy of both needles in the study population

\begin{tabular}{lllll}
\hline In all cases & \multicolumn{2}{l}{ Final diagnosis } & Total \\
\cline { 2 - 4 } & & True + ve & True - ve & \\
\hline Echo Tip Ultra HD needle & +ve & 8 & 0 & 8 \\
& $-v e$ & 13 & 1 & 14 \\
Total & & 21 & 1 & 22 \\
Echo Tip ProCore HD needle & +ve & 6 & 0 & 6 \\
& $-v e$ & 15 & 1 & 16 \\
Total & & 21 & 1 & 22 \\
\hline
\end{tabular}

Echo Tip Ultra HD needle: sensitivity (\%), 38\%; specificity (\%), 100\%; positive predictive value $\left(\mathrm{PV}^{+}\right)(\%), 100 \%$; negative predictive value $\left(\mathrm{PV}^{-}\right)(\%), 7.14 \%$; false negative rate (\%), 61.90\%; diagnostic accuracy (\%), 40.9\%

Echo Tip ProCore HD needle: sensitivity (\%), $28.57 \%$; specificity (\%), $100 \%$; positive predictive value $\left(\mathrm{PV}^{+}\right)(\%), 100 \%$; negative predictive value $\left(\mathrm{PV}^{-}\right)(\%)$, $6.25 \%$; false negative rate (\%), $71.42 \%$; diagnostic accuracy $(\%)=31.81 \%$

ultrasound images of the target lymph node or mediastinal lesion by (Hitachi EUB 7000 ultrasound) were taken through a direct contact technique and ultrasonographic characteristics was determined and recorded prior to biopsy. The needle was then advanced through the bronchoscope channel $(2 \mathrm{~mm})$, in the bronchoscope the needle is encased in an internal sheath that protects the working channel of the bronchoscope. A stylet was present inside the hollow needle at the time of insertion to clear any bronchial or cartilage plugs that may be collected when traversing the tracheal wall. The external surface of the needle is grooved at the distal end rendering it more hyperechoic and thus improving ultrasound visualization. The outer plastic sheath was advanced until its tip appears on the white light bronchoscope screen, then the whole bronchoscope navigates in the tracheobronchial tree to the site of the targeted extrabronchial lesion to select the safest tract, the largest diameter of the lesion and avoid highly vascular or necrotic areas for TBNA. At that site the needle was advanced under direct ultrasound guidance into the lesion. Immediately after puncturing the lymph node or mediastinal mass lesion stylet was removed and negative suction was applied using vaclok syringe or EBUS-TBNA capillary sampling technique, punctures were performed once by Echo Tip Ultra HD 22G needle and, once by Echo Tip ProCore HD 22G needle by hub against wall or cough technique or combination of both accordingly.

Table 2 Comparison between both needles regarding the representative cellularity of the sample

\begin{tabular}{llll}
\hline $\begin{array}{l}\text { Representative } \\
\text { needle }\end{array}$ & $\begin{array}{l}\text { Echo Tip Ultra HD } \\
\text { needle }\end{array}$ & $\begin{array}{l}\text { Echo Tip ProCore HD } \\
\text { needle }\end{array}$ & $p$ value \\
\hline Yes & 18 & 10 & 0.012 \\
No & 4 & 12 & \\
Total & 22 & 22 & \\
\hline
\end{tabular}


Table 3 Sensitivity, specificity, predictive value, and diagnostic accuracy of both needles in malignant cases

\begin{tabular}{|c|c|c|c|c|c|}
\hline \multirow[t]{2}{*}{ In malignant cases } & & \multicolumn{3}{|c|}{ Final diagnosis } & Total \\
\hline & & \multicolumn{2}{|c|}{ True +ve } & True -ve & \\
\hline \multirow[t]{2}{*}{ Echo Tip Ultra HD needle } & + ve & \multicolumn{2}{|l|}{4} & 0 & 4 \\
\hline & $-v e$ & \multicolumn{2}{|l|}{4} & 0 & 4 \\
\hline Total & & \multicolumn{2}{|l|}{8} & 0 & 8 \\
\hline \multirow[t]{2}{*}{ Echo Tip ProCore HD needle } & $+v e$ & \multicolumn{2}{|l|}{2} & 0 & 2 \\
\hline & -ve & \multicolumn{2}{|l|}{6} & 0 & 6 \\
\hline \multicolumn{2}{|l|}{ Total } & \multicolumn{2}{|l|}{8} & 0 & 8 \\
\hline \multirow[t]{2}{*}{ In malignant cases } & & \multicolumn{2}{|c|}{ Echo Tip Ultra HD needle diagnosis } & Total & $p$ value \\
\hline & & Yes & No & & \\
\hline \multirow[t]{2}{*}{ Echo Tip ProCore HD needle diagnosis } & Yes & 2 & 0 & 2 & \multirow[t]{2}{*}{0.3016} \\
\hline & No & 2 & 4 & 6 & \\
\hline Total & & 4 & 4 & 8 & \\
\hline
\end{tabular}

Echo Tip Ultra HD needle: sensitivity (\%), 50\%; positive predictive value $\left(\mathrm{PV}^{+}\right)(\%), 100 \%$; diagnostic accuracy (\%), 50\%

Echo Tip ProCore HD needle: sensitivity (\%), 25\%; positive predictive value (PV $\left.{ }^{+}\right)(\%), 100 \%$; diagnostic accuracy $(\%), 25 \%$

\section{Cytological examination of TBNA specimens}

After each needle pass, the needle was withdrawn and a small amount of material was applied to a slide. Smears were prepared by laying one slide over another, permitting the weight of the upper slide to spread the material, and pulling the slides apart horizontally which were immediately alcohol fixed in 95\% ethyl alcohol for Papanicolaou and hematoxylin and eosin stains [6].

\section{Statistical analysis}

Data were statistically described in terms of mean \pm standard deviation $( \pm S D)$ and range or frequencies (number of cases) and percentages when appropriate. A comparison between 2 needles was done using the
McNemar test. $p$ values less than 0.05 was considered statistically significant. All statistical calculations were done using the computer program IBM SPSS (Statistical Package for the Social Science; IBM Corp, Armonk, NY, USA) release 22 for Microsoft Windows.

\section{Results}

Twenty-four patients with mediastinal lesions were enrolled in the study. Out of them, 2 patients were excluded as they discovered to have mediastinal cyst by CP-EBUS, and this was confirmed by chest MRI. The 22 patients who had negative ultrasound screening for peripheral lymphadenopathy consequently underwent CPEBUS-TBNA using the Echo Tip Ultra HD 22G and

Table 4 Sensitivity, specificity, predictive value, and diagnostic accuracy of both needles in non-malignant cases

\begin{tabular}{|c|c|c|c|c|}
\hline \multirow[t]{2}{*}{ In non-malignant cases } & & \multicolumn{2}{|c|}{ Final diagnosis } & \multirow[t]{2}{*}{ Total } \\
\hline & & True +ve & True -ve & \\
\hline \multirow[t]{2}{*}{ Echo Tip Ultra HD needle } & +ve & 4 & 0 & 4 \\
\hline & -ve & 9 & 1 & 10 \\
\hline Total & & 13 & 1 & 14 \\
\hline \multirow[t]{2}{*}{ Echo Tip ProCore HD needle } & +ve & 4 & 0 & 4 \\
\hline & $-v e$ & 9 & 1 & 10 \\
\hline Total & & 13 & 1 & 14 \\
\hline \multirow[t]{2}{*}{ In non-malignant cases } & & \multicolumn{2}{|c|}{ Echo Tip Ultra HD needle diagnosis } & Total \\
\hline & & Yes & No & \\
\hline \multirow[t]{2}{*}{ Echo Tip ProCore HD needle diagnosis } & $+v e$ & 3 & 2 & 5 \\
\hline & -ve & 2 & 7 & 9 \\
\hline Total & & 5 & 9 & 14 \\
\hline$p$ value & & 0.9102 & & \\
\hline
\end{tabular}

Echo Tip Ultra HD and Echo Tip ProCore HD needles: sensitivity (\%), 30.76\%; specificity (\%), 100\%; positive predictive value (PV ${ }^{+}$) (\%), 100\%; negative predictive value $\left(\mathrm{PV}^{-}\right)(\%), 10 \%$; false negative rate $(\%), 69.23 \%$; diagnostic accuracy $(\%), 35.71 \%$ 

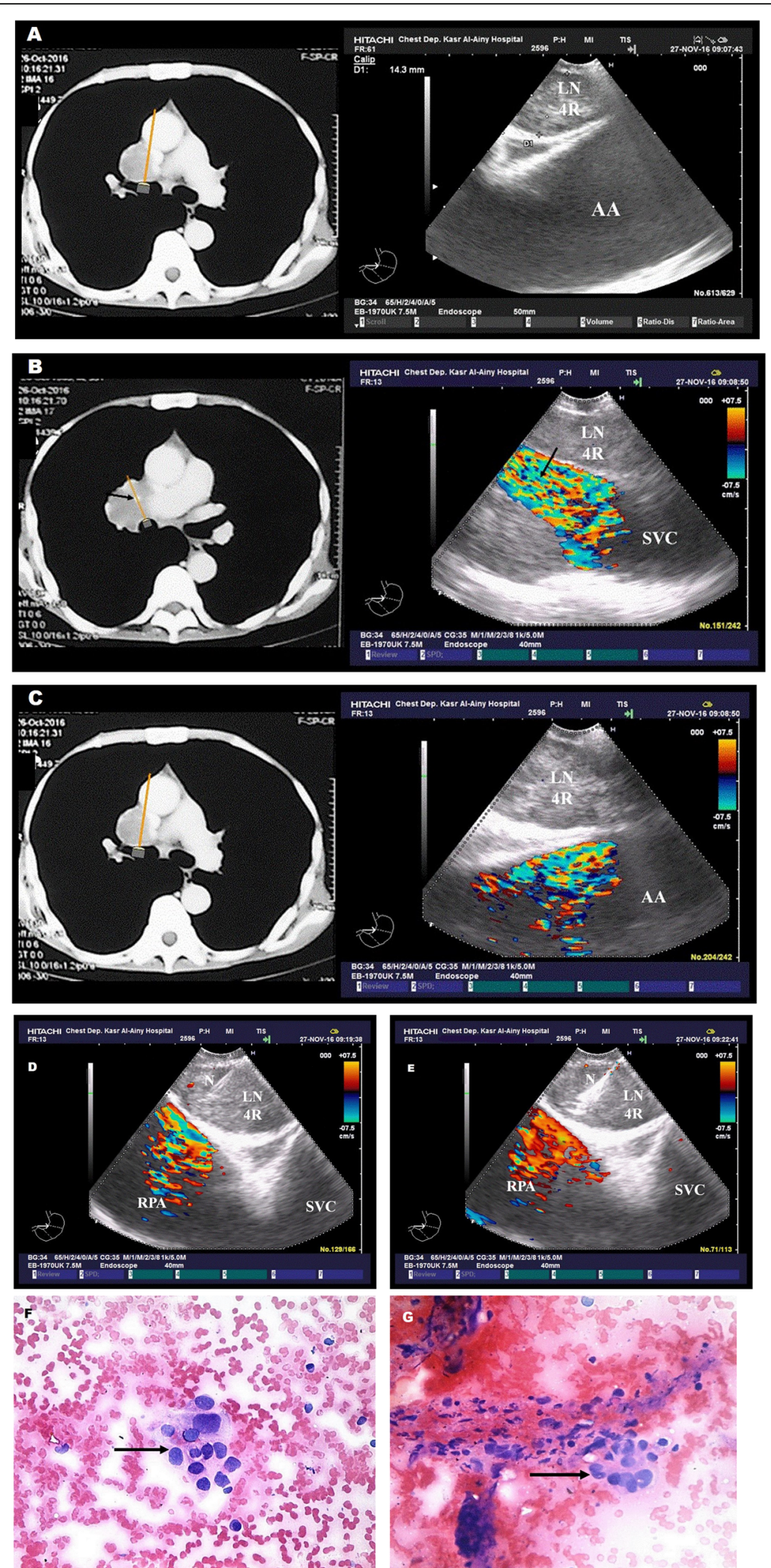

Fig. 1 (See legend on next page.) 
(See figure on previous page.)

Fig. 1 a CT chest mediastinal window showing enlarged right lower paratracheal lymph node (4R). The site of the CP-EBUS bronchoscope and direction of ultrasound view was illustrated. The EBUS ultrasound image shows enlarged right lower paratracheal lymph node (4R) and the ascending aorta (AA). $\mathbf{b}$ CT chest mediastinal window showing enlarged 4R lymph node with marked compression on the superior vena cava (SVC) (black arrow). The EBUS ultrasound color Doppler image showing enlarged 4R lymph node and the SVC with site of compression (black arrow). c CT chest mediastinal window showing enlarged 4R lymph node. The EBUS ultrasound color Doppler image shows enlarged 4R lymph node and the ascending aorta. $\mathbf{d}$ Ultrasound color Doppler image shows Echo Tip ProCore HD 22G needle (N) passing through enlarged lower paratracheal lymph node (4R), right pulmonary artery (RPA), and S.V.C. e Ultrasound color Doppler image shows Echo Tip Ultra HD $22 \mathrm{G}$ needle passing through enlarged right lower paratracheal lymph node (4R), RPA, and S.V.C. f Echo Tip Ultra HD 22G EBUS-TBNA needle sample shows adenocarcinoma (black arrow) (H\&E, × 400). g Echo Tip ProCore HD 22G EBUS-TBNA needle sample shows adenocarcinoma (black arrow)

Echo Tip ProCore HD 22G needles. Their mean age was 52.2 years. Males were $60 \%$, while females were $40 \%$.

CP-EBUS-TBNA diagnosed 11 patients with diagnostic accuracy $50 \%$, sensitivity $47.6 \%$, specificity $100 \%$, positive predictive value $100 \%$, and negative predictive value $9 \%$ with a false negative rate $52.38 \%$. The Echo Tip Ultra HD needle diagnosed 9 patients with diagnostic accuracy $40.9 \%$, sensitivity $38 \%$, specificity $100 \%$, positive predictive value $100 \%$, and negative predictive value $7.14 \%$ with a false negative rate $61.9 \%$ compared to the Echo Tip ProCore HD needle, which diagnosed 6 patients with diagnostic accuracy $31.8 \%$, sensitivity $28.5 \%$, specificity $100 \%$, positive predictive value $100 \%$, and negative predictive value $6.25 \%$ with a false negative rate $71.42 \%$, and there is no statistically significant difference between the 2 needles (Table 1 ).

The Echo Tip Ultra 22G HD needle had more representative cellularity in 18 cases compared to the Echo Tip ProCore 22G HD needle, which was representative in 10 cases with a significant $p$ value (Table 2). Eight patients were malignant cases ( 5 cases as metastatic adenocarcinoma, 2 cases as lymphoma, 1 case as squamous cell carcinoma); CPEBUS TBNA diagnosed 4 cases with 50\% diagnostic accuracy, sensitivity $50 \%$, and $100 \%$ positive predictive value. These 4 cases were 2 metastatic adenocarcinoma, 1 squamous cell carcinoma, and another 1 case as lymphoma. The Echo Tip Ultra HD needle diagnosed the 4 cases forming diagnostic accuracy $50 \%$ with sensitivity $50 \%$ and positive predictive value $100 \%$. Two cases were diagnosed by Echo Tip ProCore HD needle forming diagnostic accuracy $25 \%$ with sensitivity $25 \%$ and positive predictive value $100 \%$ with no statistically significant difference between the 2 needles (Table 3). Fourteen patients were non-malignant cases; CP-EBUS TBNA diagnosed 7 cases with diagnostic accuracy $50 \%$, sensitivity $46.15 \%, 100 \%$ specificity, positive predictive value $100 \%$, and negative predictive value $12.5 \%$. Both needles diagnosed 5 cases forming diagnostic accuracy $35.7 \%$ with sensitivity $30.76 \%$, specificity $100 \%$, positive predictive value $100 \%$, and negative predictive value $10 \%$ with a false negative rate $69.23 \%$ (Table 4).

\section{Discussion}

Mediastinal lymphadenopathy, peribronchial, and paratracheal lesions are challenging situations owing to the diversity of etiologies and the difficulty in obtaining biopsy from these regions [7]. Mediastinoscopy has been the primary diagnostic techniques for biopsy. Nevertheless, EBUS-TBNA has gained popularity because it is minimally invasive and enables real-time TBNA [3]. The High-Definition Echo Tip ProCore needle is a 22-gauge beveled needle, allowing for core biopsy along with aspiration material; this additional histological sampling could improve the yield of EBUS-TBNA [8]. Consequently, the aim of this study was to compare between Echo Tip ProCore HD-EBUS 22G and Echo Tip Ultra HD-EBUS 22G needles in the diagnosis of mediastinal lesions using CP-EBUS.

In the present study, the mean age of the study population was $52.2 \pm 12.59$ years. Nearly similar results were documented by Nagarthinam et al., Manos et al., and Desai et al. [9-11]. Regarding the sex distribution, male predominance was observed representing $60 \%$ of the study population; these findings disagree with Manos et al. [10] who observed female predominance representing $67 \%$ of the study population.

In our study, it was found that CP-EBUS TBNA diagnosed 11 patients forming diagnostic accuracy 50\% with sensitivity $47.6 \%$, specificity $100 \%$, positive predictive value $100 \%$, and negative predictive value $9 \%$ with a false negative rate $52.38 \%$. This disagreed with Zambon et al. [12] who found that EBUS-TBNA reached a diagnosis in 17 out of 19 cases forming diagnostic accuracy 89\% [12]. The most plausible explanation of lower diagnostic accuracy in our study could be due to inadequate crushed cells obtained by the Echo Tip ProCore HD needle, which was inadequate for histopathological examination.

The Echo Tip Ultra HD needle diagnosed 9 patients forming diagnostic accuracy $40.9 \%$ with sensitivity $38 \%$, specificity $100 \%$, positive predictive value $100 \%$, and negative predictive value $7.14 \%$ with a false negative rate 61.9\% compared to Echo Tip ProCore HD needle which diagnosed in 6 patients forming diagnostic accuracy $31.8 \%$ with sensitivity $28.5 \%$, specificity $100 \%$, positive predictive value $100 \%$, and negative predictive value $6.25 \%$ with a false negative rate $71.42 \%$ with no statistically significant difference between the 2 needles; these 

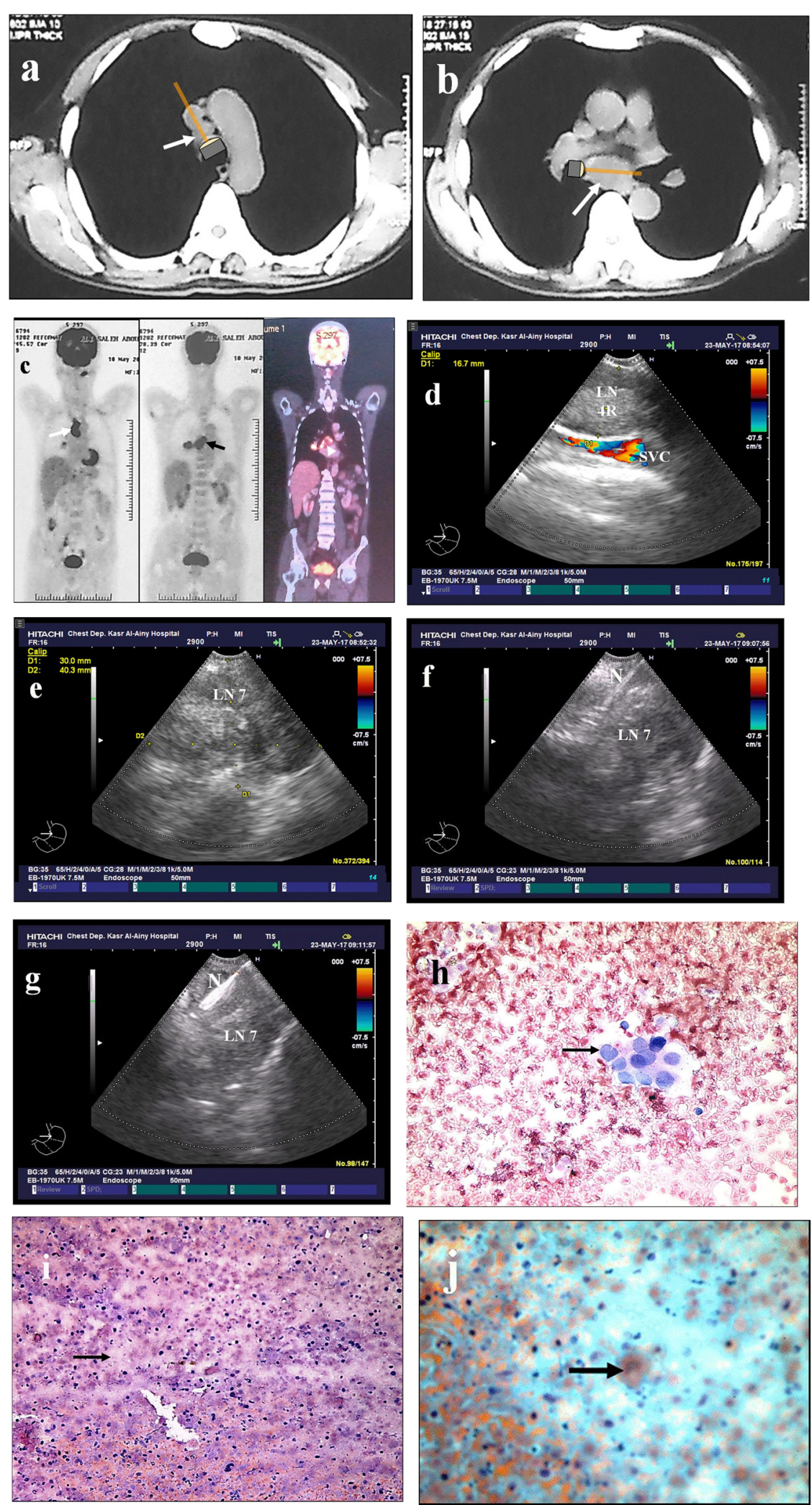

Fig. 2 (See legend on next page.) 


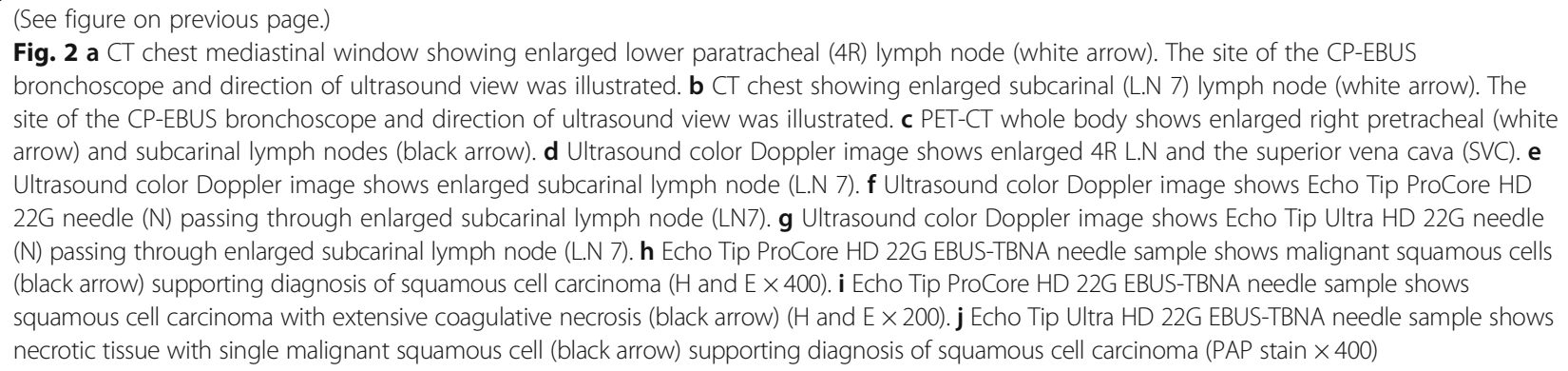

findings were lower than that recorded by Zambon et al. [11] who found that Echo Tip Ultra HD needle reached diagnosis in 14 out of 19 cases forming diagnostic accuracy $73.6 \%$ and Echo Tip ProCore HD needle reached diagnosis in 16 out of 19 cases forming diagnostic accuracy $84.2 \%$. Again, these findings disagreed with Nagarathinam et al. [9] where definitive diagnosis was reached in 16 out of 19 patients by Echo Tip ProCore HD needle forming diagnostic yield of $84.21 \%$. The divergence of diagnostic accuracy can be attributed to the use of immunohistochemistry workup which was performed in 13 (68.42\%) tissue core specimens obtained by the Echo Tip ProCore HD needle and led to a definitive diagnosis.

Desai et al. [11] used the 22G Echo Tip ProCore needle in 17 patients; 18 lymph node stations were sampled, 9 from the subcarinal lymph node (50\% of patients), 6 from the interlobar lymph node (33\%), and 3 from the lobar lymph node (17\%). Diagnosis was achieved in $89 \%$ of patients: sarcoidosis in 12/16 patients and malignancy in $4 / 16$ patients.

Regarding cellularity of obtained specimens, in our study it, was found that the Echo Tip Ultra 22G HD needle had more representative cellularity in 18 out of 22 cases (82\%) compared to the Echo Tip ProCore 22G HD needle which was representative in 10 cases out of 22 cases $(45 \%)$ with a significant $p$ value between the 2 needles. Witt et al. [13] compared EUS-FNA using the Echo Tip Ultra 22G HD and Echo Tip ProCore 22G HD needles in 18 cases. They reported that the Echo Tip ProCore 22G HD needle affords similar cytologic interpretability, diagnostic accuracy, and amount of cell block material as the Echo Tip Ultra 22G HD needle. Xing et al. [14] concluded that the Echo Tip ProCore HD needle does not provide additive value when performing EBUS-TBNA of the mediastinal or hilar lymph nodes regarding specimen adequacy.

In the present study, it was found that out of the 22 patients of the study population, 8 patients were malignant cases; CP-EBUS-TBNA diagnosed 4 cases forming diagnostic accuracy $50 \%$ compared to other diagnostic modalities with sensitivity $50 \%$ and positive predictive value $100 \%$. Malignant diagnoses included 2 metastatic adenocarcinoma (Fig. 1), 1 large cell lymphoma, and 1 squamous cell carcinoma (Fig. 2). The Echo Tip Ultra HD needle diagnosed the 4 cases forming diagnostic accuracy $50 \%$ with sensitivity $50 \%$ and positive predictive value $100 \%$, and only 2 cases were diagnosed by Echo Tip ProCore HD needle forming diagnostic accuracy $25 \%$ with sensitivity $25 \%$ and positive predictive value $100 \%$ with no statistically significant difference between the 2 needles. Conversely, this disagreed with Sivokozov [15] who performed five procedures using both needles in cases highly suspicious for lymphoma. In all cases, a final diagnosis was established, but only in two cases using the Echo Tip Ultra HD needle, whereas the ProCore showed definite efficacy for all the patients.

These findings were lower than that mentioned by Nagarathinam et al. [9] who have diagnosed 10 malignant patients out of 18 cases. Malignant diagnoses in his study included 2 small cell carcinoma, 1 pulmonary adenocarcinoma, 2 metastatic breast carcinoma, 3 metastatic renal cell carcinoma, 1 metastatic colonic adenocarcinoma, and 1 poorly differentiated carcinoma.

Similar findings were recorded by Desai et al. [11] who had diagnosed 4 malignant patients out of 16 cases. Malignant diagnoses included squamous cell carcinoma, adenocarcinoma, and mesothelioma, and none had lymphoma.

In the present study, 14 patients were non-malignant cases; CP-EBUS-TBNA was diagnostic in 7 cases with diagnostic accuracy 50\% compared to other diagnostic modalities, sensitivity $46.15 \%$, specificity $100 \%$, positive predictive value $100 \%$, and negative predictive value $12.5 \%$. Non-malignant diagnoses included 6 sarcoidosis cases and 1 fibrosing mediastinitis case which was confirmed by mediastinoscopy. Both needles were diagnostic in 5 cases forming diagnostic accuracy $35.7 \%$ with sensitivity $30.76 \%$, specificity $100 \%$, positive predictive value $100 \%$, and negative predictive value $10 \%$ with a false negative rate $69.23 \%$. Higher findings were recorded by Desai et al. [11] who diagnosed 12 out of 16 non-malignant patients with Echo Tip ProCore HD needle, and all cases were diagnosed as sarcoidosis but with negative needle aspirates and positive needle core biopsy. 
In conclusion, the Echo Tip ProCore needle was designed to collect the cytology and core biopsy specimens at the needle tip and the reverse-beveled side of the needle, respectively. Accordingly, core tissue samples will allow practitioners to fewer passes and less need to consider surgical sampling [16]. However, in this study, the Echo Tip ProCore needle-diagnosed cases were dependent upon cytological sampling and not on the core biopsy specimen which was small and moreover crushed and inadequate for diagnosis.

\section{Abbreviations}

HD-EBUS 22G: High-definition endobronchial ultrasound 22 gauge; CPEBUS: Convex probe endobronchial ultrasound; TBNA: Transbronchial needle aspiration; CT: Computed tomography; MRI: Magnetic resonance imaging; PET/CT: Positron emission tomography/computed tomography; EBUSTBNA: Endobronchial ultrasound transbronchial needle aspiration; EUSFNS: Endoscopic ultrasound fine needle aspiration

\section{Acknowledgements}

The authors thank all patients for the use of their data in this research. The authors also thank our colleagues in the Chest Diseases Department, Cairo University, for their assistance.

\section{Authors' contributions}

All authors have contributed actively in the work, and the manuscript has been read and approved by all the authors for submission. SA Hussein: study conception or design, data acquisition and analysis, doing EBUS-TBNA, interpretation of data, manuscript drafting, revision, and final approval. AO Shalaby: study conception or design, manuscript drafting, revision, and final approval. KM Kamel: study conception or design, doing EBUS-TBNA, data analysis and interpretation, manuscript drafting, revision, and final approval. AA Hareedy: interpretation of TBNA samples, manuscript drafting, revision, and final approval. HM Abdelhamid: data acquisition manuscript drafting, revision, and final approval.

\section{Funding}

This research did not receive any specific grant from funding agencies in the public, commercial, or not-for-profit sectors.

\section{Availability of data and materials}

The datasets used and/or analyzed during the current study are available from the corresponding author on reasonable request.

\section{Ethics approval and consent to participate}

The research ethics committee of Chest Department, Kasr Al Ainy, Cairo University, has approved this study, but the reference number is not applicable and not available. Written informed consent was obtained from all study participants before enrollment in the study.

\section{Consent for publication}

A written informed consent was obtained from the patients for the publication of their details.

\section{Competing interests}

The authors declare no financial of non-financial competing interest.

\section{Author details}

${ }^{1}$ Chest Department, Faculty of Medicine, Cairo University, Giza, Egypt.

${ }^{2}$ Pathology Department, Faculty of Medicine, Cairo University, Giza, Egypt.

Received: 9 March 2020 Accepted: 8 April 2020

Published online: 13 May 2020

\section{References}

1. Chen C, Mu C, Su M, Mao J, Zhu Y, Huang J (2017) Endobronchial ultrasound-guided transbronchial needle aspiration increases the yield of transbronchial lung biopsy for the evaluation of peribronchial lesions. Chin Med J 130(1):11
2. Dietrich C, Annema J, Clementsen P, Cui X, Borst M, Jenssen C (2015) Ultrasound techniques in the evaluation of the mediastinum, part l: endoscopic ultrasound (EUS), endobronchial ultrasound (EBUS) and transcutaneous mediastinal ultrasound (TMUS), introduction into ultrasound techniques. Journal of thoracic disease 7(9):311

3. Sun W, Qian Q, Xiao X, Song Y (2017) Application value of endobronchial ultrasound-guided transbronchial needle aspiration in the diagnosis and treatment of mediastinal and pulmonary diseases. Transl Surg 2:6-9

4. Vilmann P, Clementsen P, Colella S, Siemsen M, De Leyn P, Dumonceau J et al (2015) Combined endobronchial and esophageal endosonography for the diagnosis and staging of lung cancer: European Society of Gastrointestinal Endoscopy (ESGE) Guideline, in cooperation with the European Respiratory Society (ERS) and the European Society of Thoracic Surgeons (ESTS). Endoscopy. 47(6):545-559

5. VanderLaan P, Wang H, Majid A, Folch E (2014) Endobronchial ultrasoundguided transbronchial needle aspiration (EBUS-TBNA): an overview and update for the cytopathologist. Cancer cytopathology 122(8):561-576

6. Solomides C, Johnston W, Elson C. Respiratory tract. In: Comprehensive cytopathology (eds. Bibbo, M. and Wilbur, D.). Elsevier, London. 2015

7. Chen Y, Jiang J, Mao J, Huang (2016) Diagnostic value of endobronchial ultrasound-guided transbronchial needle aspiration (EBUS-TBNA) in solitary mediastinal, hilar lymphadenectasis, or peribronchial lesions: six cases reports and review of literature. Medicine 95(44):5249

8. Ariza-Prota M, Bango Álvarez A, Pérez L, Pando-Sandoval A, Fuentes N, Casan P (2015) From cytology to histology: diagnosis of a relapsed mediastinal lymphoma by endobronchial ultrasound transbronchial histological needle. Respirology case reports 3(2):68-71

9. Nagarathinam R, Russell D, Nead M, Giampoli E (2014) Endobronchial Ultrasound Guided Transbronchial Core Needle Biopsy (CNB) and Fine Needle Aspiration (FNA) Using Echotip Procore ${ }^{\mathrm{TM}}$ Needle versus Conventional Needle-A Retrospective Comparison of Diagnostic Utility. Journal of the American Society of Cytopathology 3(5):67

10. Manos S, Monaco S, Wilson D, Pantanowitz L (2014) Endobronchial ultrasound-guided (EBUS) Procore sampling: comparison to fine needle aspiration for mediastinal lymph nodes. Journal of the American Society of Cytopathology 3(5):84

11. Desai N, Greenhill S, Vance M, Kovitz L (2012) Endobronchial ultrasound guided transbronchial core needle biopsy using a novel needle. Am J Respir Crit Care Med 185:A1105

12. Zambon M, Nagarathinam R, Russell D, Giampoli E, Nead M (2015) ProCore needles versus standard 22-gauge needle in EBUS guided biopsies. Chest. 148(4):791

13. Witt B, Adler D, Hilden K, Layfield L (2013) A comparative needle study: EUSFNA procedures using the HD ProCore ${ }^{T M}$ and EchoTip ${ }^{\oplus}$ 22-gauge needle types. Diagn Cytopathol 41(12):1069-1074

14. Xing J, Manos S, Monaco S, Wilson D, Pantanowitz L (2016) Endobronchial ultrasound-guided transbronchial needle aspiration: a pilot study to evaluate the utility of the ProCore biopsy needle for lymph node sampling. Acta Cytol 60(3):254-259

15. Sivokozov I (2014) First experience with the 22 gauge Echo Tip ProCore EBUS needle in cases with suspected lymphoma. The Channel 2:14-15

16. Dincer H, Andrade R, Zamora F, Podgaetz E (2016) A new needle on the block: Echo Tip ProCore endobronchial ultrasound needle. Medical Devices 9:467-473

\section{Publisher's Note}

Springer Nature remains neutral with regard to jurisdictional claims in published maps and institutional affiliations. 